\title{
PALLAS: Planetary Analogues Laboratory for Light, Atmosphere, and Surface Simulations
}

\section{I.L. ten Kate* \& M. Reuver}

Earth Sciences Department, Utrecht University, Utrecht, the Netherlands

* Corresponding author. Email: i.l.tenkate@uu.nl

Manuscript received: 17 0ctober 2014, accepted: 26 April 2015

\section{Abstract}

Humankind has been interested in space throughout the ages and studies of the universe and our own solar system have been ongoing since the first observations of celestial bodies. In the current era space exploration has provided in situ data for the different bodies in our solar system. To fully comprehend the underlying processes occurring in these bodies, missions and telescope observations are, however, not sufficient and additional modelling studies, both numerical and analogue, are necessary. In this paper we present a new facility specifically designed to experimentally study organic compounds under simulated planetary (sub)surface conditions on rocky bodies in our solar system: PALLAS, the Planetary Analogues Laboratory for Light, Atmosphere, and Surface Simulations. We give an overview of planetary conditions that can be simulated in this facility and that are known to affect organic compounds: radiation, atmospheric composition, temperature and surface composition.

Keywords:: laboratory simulations, planetary environments, organic molecules, organic-mineral interactions

\section{Introduction}

Humankind has been interested in space throughout the ages and studies of the universe and our own solar system have been ongoing since the first observations of celestial bodies. In the current era space exploration has provided in situ data for the different bodies in our solar system. As well as images, a wide range of atmospheric and geoscientific data, including geological features, mineralogical composition, interior composition, presence of surface and subsurface water, and atmospheric composition and chemistry, have been returned by both orbiting and landing missions. To fully comprehend the underlying processes occurring in these bodies, missions and telescope observations are, however, not sufficient and additional modelling studies, both numerical and analogue, are necessary.

A large number of analogue simulation facilities capable of mimicking planetary surface and astrobiological conditions exists worldwide (ten Kate \& Motamedi, 2015). It is practically impossible to simulate all conditions on a solar system body in one facility, therefore different facilities focus on different aspects.
In this paper we focus on a facility designed to study experimentally organic compounds on rocky bodies in our solar system. Organic compounds are nearly all molecules containing carbon, apart from a few exceptions, for example the atmospheric gases $\mathrm{CO}$ and $\mathrm{CO}_{2}$, and have been detected throughout the universe (Ehrenfreund et al., 2011). Although the name suggests otherwise not all organic compounds are produced by living organisms. Life uses four major types of organic compounds: carbohydrates, lipids, proteins and nucleic acids (DNA and RNA). These types are themselves built up from smaller organic molecules, such as sugars, polysaccharides, fatty acids, amino acids and nucleobases. Some of these organic molecules are thought to have played a role in the origin of life and are therefore referred to as prebiotic molecules. A wide variety of organic compounds are formed and have been observed in space. Large organic compounds, such as polycyclic aromatic hydrocarbons (PAHs), are readily formed in the interstellar medium (Frenklach \& Feigelson, 1989; Parker et al., 2012). PAHs also been have been linked to processes playing a role in the origin of life (Groen et al., 2012). Smaller, more complex organic compounds are formed 
Table 1. Selected surface and atmospheric parameters of selected solar system bodies.

\begin{tabular}{|c|c|c|c|c|}
\hline Solar system body & Mercury $^{b}$ & Earth & Mars $^{c}$ & Moon \\
\hline Mass $\left(10^{24} \mathrm{~kg}\right)^{\mathrm{a}}$ & 0.33 & 5.97 & 0.64 & 0.07 \\
\hline Radius $(\mathrm{km})^{\mathrm{a}}$ & 2439.7 & 6378.14 & 3396.2 & 1738.1 \\
\hline Density $\left(\mathrm{g} \mathrm{cm}^{-3}\right)^{\mathrm{a}}$ & 5.43 & 5.52 & 3.93 & 3.35 \\
\hline Surface gravity $\left(\mathrm{m} \mathrm{s}^{-2}\right)^{\mathrm{a}}$ & 3.70 & 9.80 & 3.71 & 1.62 \\
\hline Temperature $(\mathrm{K})^{\mathrm{a}}$ & $100-700$ & $184-330$ & $130-308$ & $100-390$ \\
\hline Escape velocity $\left(\mathrm{km} \mathrm{s}^{-1}\right)^{\mathrm{a}}$ & 4.25 & 11.18 & 5.03 & 2.38 \\
\hline Length of day $(h)^{a}$ & 4222.6 & 24.0 & 24.7 & 708.7 \\
\hline Atmospheric pressure (mbar) & $10^{-11}$ & 1000 & 10 & $10^{-9}$ (day) to $10^{-12}$ (night) \\
\hline \multirow[t]{5}{*}{ Atmospheric composition } & $42 \%$ oxygen (molecular) & $78.08 \%$ nitrogen & $95.32 \%$ carbon dioxide & Argon \\
\hline & $29 \%$ sodium & $20.95 \%$ oxygen & $2.7 \%$ nitrogen & Helium \\
\hline & $22 \%$ hydrogen & $0.93 \%$ argon & $1.6 \%$ argon & Sodium \\
\hline & $6 \%$ helium & $0.036 \%$ carbon dioxide & $0.13 \%$ oxygen & Hydrogen \\
\hline & traces $\mathrm{Na}, \mathrm{K}, \mathrm{Ca}, \mathrm{Mg}$ & $\begin{array}{l}\sim 1 \% \text { water vapour } \\
\quad \text { (varies with climate) }\end{array}$ & $\begin{array}{l}0.08 \% \text { carbon monoxide } \\
0.03 \% \text { water vapor }\end{array}$ & Potassium \\
\hline
\end{tabular}

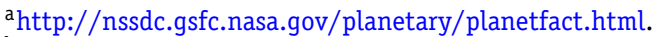

${ }^{b}$ Broadfoot et al. (1974), Shemansky \& Broadfoot (1977), Potter \& Morgan (1985, 1986), Vasavada et al. (1999), Bida et al. (2000), McClintock et al. (2009).

${ }^{\mathrm{c}}$ Catling (2009).

during the evolution of protoplanetary disks (Ciesla \& Sandford, 2012). Mechanisms include irradiation of interstellar ices (Bernstein et al., 2002), aqueous alteration of a protoplanet or meteorite (Zolensky \& McSween, 1988; Shock, 1990), FischerTropsch-type syntheses during, for example, collisions or shock waves (Hayatsu \& Anders., 1981), or thermal decomposition of certain minerals (McCollom, 2003). Even the simplest sugar has recently been detected in the interstellar medium (Beltrán et al., 2009). These space-based organics can be incorporated and further processed in planetary bodies, comets, meteorites and interplanetary dust particles (IDPs) during the formation of a planetary system. Nucleobases and PAHs have been detected in comets (Kissel \& Krueger, 1987), PAHs, carboxylic acids (CAs), nucleobases and amino acids have been detected in meteorites (Sephton, 2002; Pizzarello et al., 2006; Martins et al., 2008), and interplanetary dust particles also contain a wide range of organics (Flynn et al., 2008). Planets in our solar system have been constantly bombarded with large impactors, such as comets and asteroids, even as recently as 1.7 Gyr ago (Bottke et al., 2012), and with an ongoing flux of smaller impactors, such as meteorites and IDPs. However, the link between the origin of life and extraplanetary organics remains unknown. Whereas many hypotheses discuss whether these organics contribute to the origin of life, the fate of extraplanetary organics after delivery onto (early) planetary bodies is essentially unknown. A range of factors can alter the state and composition of the delivered organics (e.g. ten Kate, 2010). Here we present a new facility specifically designed to simulate planetary (sub)surface conditions that was recently commissioned at Utrecht University.

\section{The Planetary Analogues Laboratory for Light, Atmosphere, and Surface Simulations}

To study organic processes in a planetary surface scenario while recreating several of the conditions discussed in the previous section, we designed and built the Planetary Analogues Laboratory for Light, Atmosphere, and Surface Simulations (PALLAS). PALLAS is not only suitable for studies on organic compounds, but also for studying microorganisms exposed to different planetary conditions as well as other processes requiring non-terrestrial conditions, such as evaporation studies of lakes on Mars. Below we give an overview of PALLAS's specifications and show preliminary calibration data to give a feel for the capabilities of this facility. Table 1 gives an overview of selected surface and atmospheric parameters of solar system bodies whose surfaces can be simulated within PALLAS. Additionally, specific temperature, pressure and atmosphere conditions can be selected that can be extrapolated to asteroid or comet surfaces and interiors.

\section{Chamber description}

PALLAS (Fig. 1) is a $50 \times 50 \times 50 \mathrm{~cm}$ stainless steel vacuum chamber (Pfeiffer Vacuum) equipped with various ports and windows, and a large door for sample access. A differentially pumped sampling volume, the atmospheric sample chamber (ASC), is mounted onto the main chamber and connected via both a gate valve and a needle valve. The ASC is equipped with a turbo pump (Pfeiffer Vacuum Turbo HiPace 80) attached 


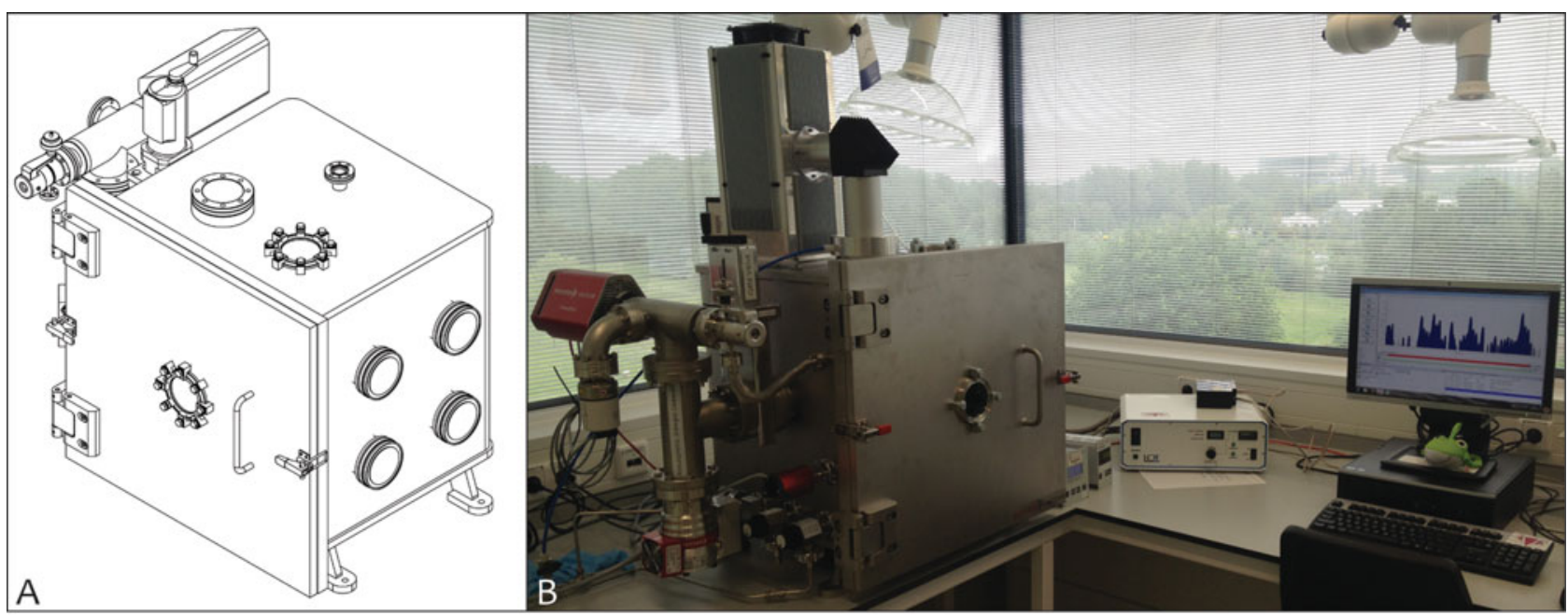

Fig. 1. PALLAS. A. A schematic drawing showing the chamber, with four side ports, two top windows, the right with a borosilicate window and the left with a UV transparent fused-silica window, and top port to mount the deuterium UV source, the main door with a borosilicate window, and mounted on the left the mass spectrometer. B. A picture showing the actual setup in the laboratory, with the atmospheric sample chamber and its mass spectrometer and turbopump, gate valve and needle valve. The solar simulator placed on top of the chamber and controlled by the supply on the right. The computer is used to monitor and log mass spectra, pressures and UV spectra. Both the lamp and the diaphragm pump are connected to the main laboratory venting system with adjustable hoods to remove ozone and gases that are pumped out of the chamber.

to a diaphragm pump (Pfeiffer Vacuum MVP 070-3), a mass spectrometer (Pfeiffer Vacuum QMG 220 M1, PrismaPlus Compact) and a pressure gauge (Pfeiffer Vacuum PKR251, 10 11 1100 mbar). The entire system (chamber + ACS) can be pumped down to pressures around $10^{-8}$ mbar through the gate valve. A xenon arc discharge lamp (LOT-Oriel, $450 \mathrm{~W}$ UV enhanced Xe, $180-900 \mathrm{~nm}$ ) is available to create the desired solar spectrum. A deuterium light source (Hamamatsu S2D2 VUV) can additionally be mounted inside the chamber to enhance the ultraviolet (UV) spectrum. The xenon lamp stands on top of the chamber and irradiates the samples through a UV transparent fused-silica window (99.5\% transmission at $193 \mathrm{~nm}$, transparent down to EUV (10 nm)). An airtight tube is mounted between the lamp housing and the fused-silica window and can be filled with $\mathrm{N}_{2}$ to minimise UV loss and ozone formation. Samples are placed on temperature-controlled tables and can be variably irradiated in the beam spot of the UV source. The intensity of the beam is measured using an 0cean 0ptics Maya2000PR0 spectrometer, optimised for the 150-400 nm wavelength range. The temperature of the sample tables is controlled using a JULAB0 FP89-AL ultra-low refrigerated heating circulator. Three gas inlet valves are connected to the chamber to insert atmospheric gases. One inlet is connected to a $\mathrm{N}_{2}$ line, which is used to vent the chamber while preventing atmospheric water from entering. Gases can be either premixed or mixed inside the chamber to obtain the desired atmospheric conditions. Atmospheric pressures inside the chamber are monitored with a pressure gauge (Pfeiffer Vacuum CMR361, 0.1-1100 mbar).

\section{Brief experimental protocol}

Samples are placed in the desired configuration on the sample tables, and then the chamber is closed and carefully pumped down through either the gate valve or the needle valve. When the pressure inside the chamber has reached the desired value (at least in the order of $10^{-7}$ mbar) a background mass spectrum is recorded. If both background and pressure requirements are met, both the gate valve and the needle valve are closed and the chamber is filled with the desired atmosphere. The chamber pressure can be monitored on the chamber pressure gauge. The ASC is continuously pumped. To carry out atmospheric analyses a little gas is let into the ACS up to pressures of around $10^{-6}$ mbar. Higher pressures may damage the mass spectrometer. Atmospheric analyses can be recorded continuously or in intervals. The sample temperature can be regulated between -90 and $+100^{\circ} \mathrm{C}$ as required for the simulated scenario.

\section{Example experiment}

PALLAS is designed to be versatile to allow a wide range of experiments. An organic-mineral interaction example experiment is described here. In this experiment selected minerals are spiked with selected organics through mechanical mixing, vapour deposition or dissolution-evaporation. The samples are analysed before being subjected to the conditions in the chamber, using non-destructive techniques including infrared and Raman spectroscopy. Samples are prepared in batches of at 


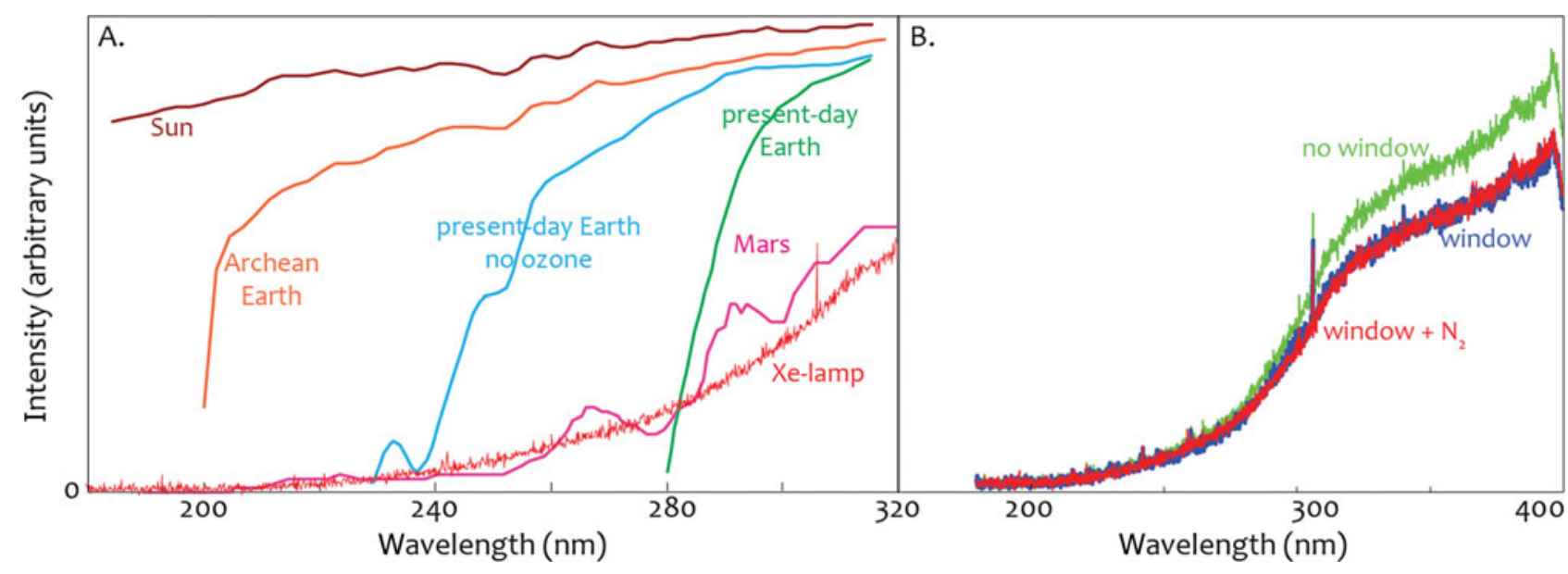

Fig. 2. The UV spectrum as received by samples in PALLAS. A. Surface scenarios: the UV spectrum at the sample location, compared to selected scenarios: the Sun, the UV flux on the Archean Earth's surface, the current Earth's surface with and without the effect of ozone and Mars' surface. Note that the spectra are plotted in arbitrary units and that the Mars UV spectrum has been scaled, to highlight the difference in the current day Mars and Earth UV scenarios. B. Effect of fused-silica window: the difference in UV intensity on the samples without the fused-silica window, with the window, and with the window and the $\mathrm{N}_{2}$ filled cylinder between the window and the lamp.

least three, one to be placed in the chamber under the UV beam, a second in the chamber in the dark and a third as a dark control outside the chamber. After the experiments the samples are analysed again using infrared and Raman spectroscopy, followed by extraction of the organics for further analysis using mass spectrometry and liquid chromatography. The mineral residue is analysed using scanning and transmission electron microscopy. Additional analysis can be carried out with secondary ion mass spectrometry (nanoSIMS) and nuclear magnetic resonance.

\section{UV radiation}

UV radiation has a very strong photodegradation effect on a wide range of organic compounds and could therefore have a sterilising effect on planetary surfaces, such as Mars (e.g. Oro \& Holzer, 1978; ten Kate et al., 2005, 2006; Stalport et al., 2009; Moores \& Schuerger, 2012). This UV photodegradation is predominantly a surface process, since UV does not penetrate a rocky surface deeper than a few 100 nanometres, depending on composition (180 $\mathrm{nm}$ has been used as a reference value (Jeong et al., 2003; Schuerger et al., 2011). Additionally, at wavelengths that are not directly damaging to organic molecules UV can have a photocatalytical effect on metal oxides (Shkrob et al., 2011), causing, for example, photo-oxidation of these organic molecules (Shkrob et al., 2010). Furthermore, photodissociation of $\mathrm{H}_{2} \mathrm{O}$ by $\mathrm{UV}$ leads to the formation of highly reactive $\mathrm{OH}$ radicals, which in turn can also react with organic compounds.

The Sun put out more UV in the early stages of the solar system (Cnossen et al., 2007; Claire et al., 2012), a factor that needs to be taken into account when simulating conditions representing the early solar system. As result of their differing atmospheric compositions, the terrestrial planets have very different ultraviolet histories (Cockell, 2000). For example, $\mathrm{CO}_{2}$ absorbs wavelengths shorter than $190 \mathrm{~nm}$, but everything longer than that will reach the surface, as is the case on Mars (Patel et al., 2002). Present-day Earth is protected from most of the damaging radiation $(<300 \mathrm{~nm}$ ) through its ozone layer, but early Earth's atmosphere did not contain any ozone and therefore more UV reached the early Earth's surface. Fig. 2A shows the UV spectrum of the Sun, the Archean Earth, present-day Earth with and without ozone layer and present-day Mars, in arbitrary units. Additionally, the spectrum of the xenon lamp is plotted to show its relation to the aforementioned spectra. Fig. 2B shows the spectrum of the lamp measured directly and through the fused-silica window with and without $\mathrm{N}_{2}$ in the air-closed connection tube. The lamp has a warm-up time of about $20 \mathrm{~min}$; a cool lamp versus a warm lamp has a difference of $\sim 15 \%$ in intensity.

\section{Atmospheric composition}

PALLAS can be used to simulate a range of atmospheric conditions. In the case of experiments involving microorganisms the atmospheric composition in the facility is particularly important. There are two constraints: the pressure (PALLAS is a low-pressure chamber, so to keep the chamber isolated from the laboratory environment the pressure inside the chamber needs to be slightly lower than ambient pressure in the laboratory ( 1000 mbar)) and the presence of corrosive gases (even though PALLAS and most of its parts are made out of stainless steel, compounds such as $\mathrm{Cl}, \mathrm{SO}_{2}$ and $\mathrm{H}_{2} \mathrm{SO}_{4}$ can have corrosive effects when applied in large amounts, and these gases are 


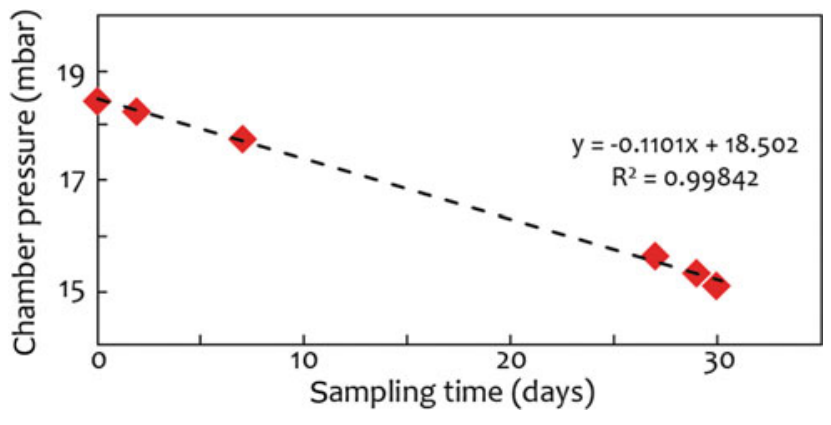

Fig. 3. Chamber pressure change during continuous sampling. During continuous sampling a tiny leak is created between the main chamber and the ASC. This leak leads to an internal pressure in the order of $10^{-6}$ mbar in the ASC and enables continuous scanning of the atmosphere in the chamber.

relatively difficult to remove only by pumping, so precautions have to be taken when simulating conditions involving these species). The vacuum inside the chamber is shown to be very stable: when filled to 10 mbar and left closed without pumping for 18 months the pressure rose $20 \%$ to 12 mbar. To monitor the atmospheric composition a small amount of gas is leaked into the ASC. Fig. 3 shows the linear drop in atmospheric pressure in the main chamber when continuously sampling gases to monitor. Here we measured a drop in pressure of about $18 \%$ over 30 days. Table 1 gives an overview of the current atmospheric composition on the Earth and Mars, whereas Fig. 4 shows a schematic evolution of the terrestrial and Martian atmospheres. This evolution is important when simulating early Earth and Mars conditions.

\section{Temperature}

The temperature on the terrestrial planets and other rocky bodies in our solar system ranges from 100 to $700 \mathrm{~K}$ (see Table 1). Not only does temperature have a large effect on the processes occurring in atmospheres, it also has a great effect on chemical reactions on the surface by, for example, enabling liquid water to exist. Simulating the full 100-700 K temperature range in a facility the size of PALLAS is very difficult. Within the scope of research that PALLAS is designed for, temperature variations between 183 and $373 \mathrm{~K}$, directly enabling Moon, Mars and early Earth simulations, are sufficient. Within this temperature range more generic conditions can be simulated that can be further extrapolated to, for example, comet and asteroid conditions using dedicated numerical models.

\section{Surface composition}

Mineral-organic interactions are important for a variety of modern geochemical phenomena and were potentially also important on extraterrestrial bodies and for the origin of life of Earth (see Cleaves et al., 2012 and references therein).

Mineral surfaces are hypothesised to play a role in protecting, selecting, concentrating, templating and catalysing reactions of prebiotic organic molecules. Well-studied minerals include clay minerals (e.g. Cairns-Smith \& Hartman, 1986), various transition metals (e.g. $\mathrm{Fe}, \mathrm{Ni}, \mathrm{Co}$ and $\mathrm{Cu}$ ), sulphide minerals, metaloxides, carbonates and olivine (Cleaves et al., 2012).

Specific processes that can be studied in PALLAS include photocatalysis, the substrate-mediated redox reactions of organics with UV and visible light by iron-rich minerals (e.g. Jia

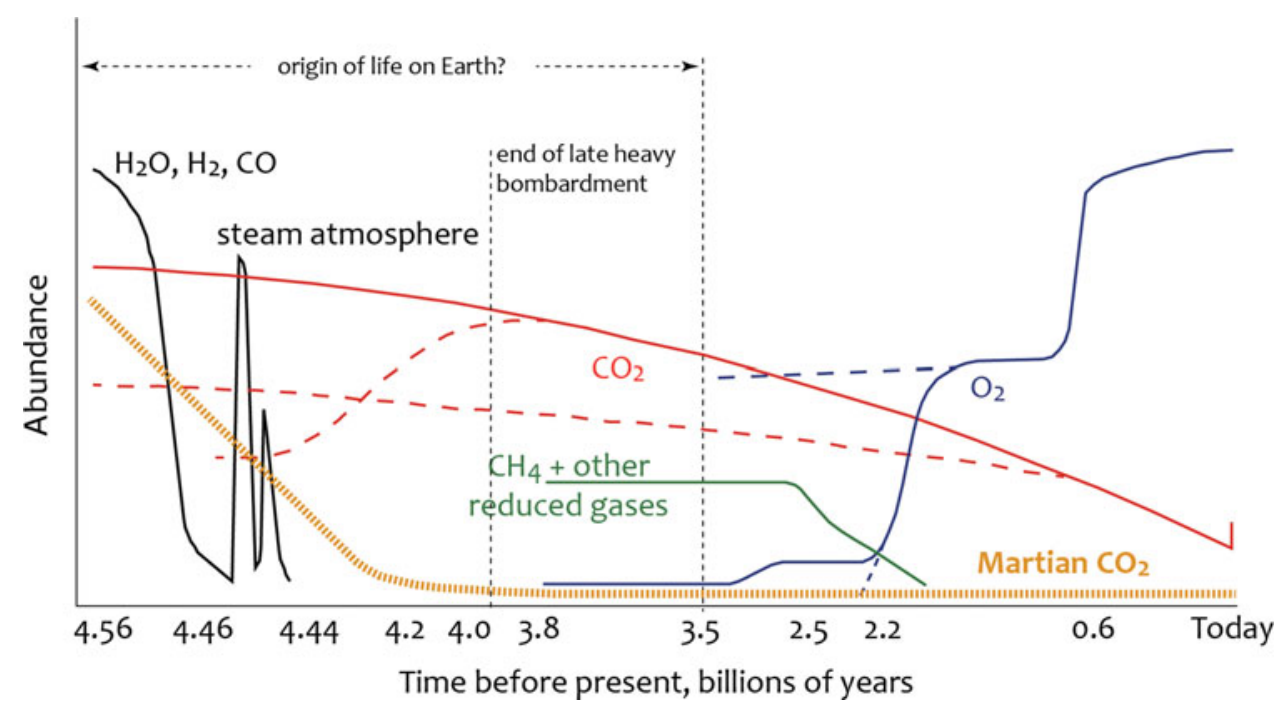

Fig. 4. Approximate evolution of the Earth's atmosphere and Martian $\mathrm{CO}_{2}$. Showing the evolution of the main terrestrial atmospheric gases as well as the main Martian atmospheric gas, $\mathrm{CO}_{2}$, as function of time. The era of heavy impacts and the window in which life on Earth originated are shown because both had a large influence on the atmospheric evolution. (Based on Ahrens, 1993; Zahnle et al., 2010; Canfield, 2005; Catling, 2009; Farquhar, 2009). 
et al., 2012) and metal-oxides (e.g. Fox \& Dulay, 1993; Shkrob et al., 2010, 2011), and aqueous alterations of minerals and mineral-organic aggregates, processes that, for example, play a role in meteorites (Shock \& Schulte, 1990).

\section{Conclusion}

Planetary environment simulation facilities are used worldwide to better understand the chemical physical processes occurring in the bodies in our solar system. PALLAS is the newest member of this family and allows us to recreate temperature, atmospheric and ultraviolet conditions on planetary surfaces. PALLAS is designed to host a wide range of research projects, including studies on organic-mineral interactions and microorganisms under extraterrestrial conditions.

\section{References}

Ahrens, T.J., 1993. Impact erosion of terrestrial planetary atmospheres. Annual Review of Earth and Planetary Sciences 21: 525-555.

Beltrán, M.T., Codella, C., Viti, S., Neri, R. \& Cesaroni, R., 2009. First detection of glycolaldehyde outside the Galactic Center. Astrophysical Journal Letters 690: L93-L96.

Bernstein, M., Dworkin, J., Sandford, S., Cooper, G. \& Allamandola, L.J., 2002. Racemic amino acids from the ultraviolet photolysis of interstellar ice analogues. Nature 416: 401-403.

Bida, T.A., Killen, R.M. \& Morgan, T.H., 2000. Discovery of calcium in Mercury's atmosphere. Nature 404: 159-161.

Bottke, W.F., Vokrouhlický, D., Minton, D., Nesvorný, D., Morbidelli, A., Brasser, R., Simonson, B. \& Levison, H.F., 2012. An Archaean heavy bombardment from a destabilized extension of the asteroid belt. Nature 485 : 78-81.

Broadfoot, A.L., Kumar, S., Belton, M.J.S. \& McElroy, M.B., 1974. Mercury's atmosphere from Mariner 10: Preliminary results. Science 185: 166169.

Cairns-Smith, A.G. \& Hartman, H., (eds), 1986. Clay minerals and the origin of life. Cambridge University Press (Cambridge).

Canfield, D.E., 2005. The early history of atmospheric oxygen: Homage to Robert A. Garrels. Annual Review of Earth and Planetary Sciences 33: 1-36.

Catling, D.C., 2009. Atmospheric evolution, Mars. In: Gornitz, V. (ed.): Encyclopedia of Paleoclimatology and Ancient Environments. Springer (Dordrecht): $66-75$.

Ciesla, F.J. \& Sandford, S.A., 2012. Organic synthesis via irradiation and warming of ice grains in the solar nebula. Science 336: 452-454.

Claire, M.W., Sheets, J., Cohen, M., Ribas, I., Meadows, V.S. \& Catling, D.C., 2012. The evolution of solar flux from $0.1 \mathrm{Nm}$ to $160 \mu \mathrm{m}$ : Quantitative estimates for planetary studies. Astrophysical Journal 757: 95.

Cleaves, H.J., II, Michalkova Scott, A., Hill, F.C., Leszczynski, J., Sahai, N. \& Hazen, $R_{.}, 2012$. Mineral-organic interfacial processes: potential roles in the origins of life. Chemical Society Reviews 41: 5502.
Cnossen, I., Sanz-Forcada, J., Favata, F., Witasse, 0., Zegers, T. \& Arnold, N.F., 2007. Habitat of early life: Solar X-ray and UV radiation at Earth's surface 43.5 billion years ago. Journal of Geophysical Research 112: E02008.

Cockell, C.S., 2000. The ultraviolet history of the terrestrial planets - implications for biological evolution. Planetary and Space Science 48: 203-214.

Ehrenfreund, P., Spaans, M. \& Holm, N.G., 2011. The evolution of organic matter in space. Philosophical Transactions of the Royal Society of London Series A -Mathematical Physical and Engineering Sciences 369: 538-554.

Farquhar, J. 2009. Atmospheric evolution, Earth. In: Gornitz, V. (ed.): Encyclopedia of Paleoclimatology and Ancient Environments. Springer (Dordrecht): 61-65.

Flynn, G.J., Keller, L.P., Wirick, S. \& Jacobsen, C., 2008. Organic matter in interplanetary dust particles. In: Kwok, S. \& Sandford, S. (eds.): Organic Matter in Space, Proceedings of the IAU Symposium No. 251. 267-275. Cambridge University Press (Cambridge).

Fox, M.A. \& Dulay, M., 1993. Heterogeneous photocatalysis. Chemical Reviews 93: 341-357.

Frenklach, M. \& Feigelson, E.D., 1989. Formation of polycyclic aromatic hydrocarbons in circumstellar envelopes. Astrophysical Journal 341: 372-384.

Groen, J.J., Deamer, D.W., Kros, A.A. \& Ehrenfreund, P.P. 2012. Polycyclic aromatic hydrocarbons as plausible prebiotic membrane components. Origins of Life and the Evolution of the Biosphere 42: 295-306.

Hayatsu, R. \& Anders, E., 1981. Organic compounds in meteorites and their origins. Topics in Current Chemistry 99: 1-37.

Jeong, I.S., Kim, J.H. \& Im, S., 2003. Ultraviolet-enhanced photodiode employing n-Zn0/p-Si structure. Applied Physics Letters 83: 2946.

Jia, H., Zhao, J., Fan, X., Dilimulati, K. \& Wang, C., 2012. Photodegradation of phenanthrene on cation-modified clays under visible light. Applied Catalysis B - Environmental 123-124: 43-51.

Kissel, J. \& Krueger, F.R., 1987. The organic component in dust from comet Halley as measured by the PUMA mass spectrometer on board Vega 1. Nature 326: 755-760.

Martins, Z., Botta, O., Fogel, M.L., Sephton, M.A., Glavin, D.P., Watson, J.S., Dworkin, J.P., Schwartz, A.W. \& Ehrenfreund, P., 2008. Extraterrestrial nucleobases in the Murchison meteorite. Astrophysical Journal 270: 130136.

McClintock, W.E., Suffix, R.J.V.S.J., Bradley, E.T., Killen, R.M., Mouawad, N., Sprague, A. L., Burger, M.H., Solomon, S.C. \& Izenberg, N.R., 2009. MESSENGER observations of Mercury's exosphere: Detection of magnesium and distribution of constituents. Science 324: 610-613.

McCollom, T.M., 2003. Formation of meteorite hydrocarbons from thermal decomposition of siderite $\left(\mathrm{FeCO}_{3}\right)$. Geochimica et Cosmochimica Acta 67: 311317.

Moores, J.E. \& Schuerger, A.C., 2012. UV degradation of accreted organics on Mars: IDP longevity, surface reservoir of organics, and relevance to the detection of methane in the atmosphere. Journal of Geophysical Research 117: E08008.

Oro, J.J. \& Holzer, G.G., 1978. The effects of ultraviolet light on the degradation of organic compounds: a possible explanation for the absence of organic matter on Mars. Life Sciences and Space Research 17: 77-86.

Parker, D.S.N., Zhang, F., Kim, Y.S., Kaiser, R.I., Landera, A., Kislov, V.V., Mebel, A.M. \& Tielens, A.G.G.M., 2012. Low temperature formation of naphthalene and its role in the synthesis of PAHs (polycyclic aromatic 
hydrocarbons) in the interstellar medium. Proceedings of the National Academy of Sciences of the United States of America 109: 53-58.

Patel, M., Zarnecki, J. \& Catling, D., 2002. Ultraviolet radiation on the surface of Mars and the Beagle 2 UV sensor. Planetary and Space Science 50: 915927.

Pizzarello, S., Cooper, G.W. \& Flynn, G.J., 2006. The nature and distribution of the organic material in carbonaceous chondrites and interplanetary dust particles. In: Meteorites and the Early Solar System II. University of Arizona Press (Tucson): 625-651.

Potter, A. \& Morgan, T.H., 1985. Discovery of sodium in the atmosphere of Mercury. Science 229: 651-653.

Potter, A.E. \& Morgan, T.H., 1986. Potassium in the atmosphere of Mercury. Icarus 67: 336-340.

Schuerger, A.C., Clausen, C. \& Britt, D.T., 2011. Methane evolution from UVirradiated spacecraft materials under simulated Martian conditions: Implications for the Mars Science Laboratory (MSL) mission. Icarus 213: 393403.

Sephton, M., 2002. Organic compounds in carbonaceous meteorites. National Product Report, the Royal Society of Chemistry 19: 292-311.

Shemansky, D.E. \& Broadfoot, A.L., 1977. Interaction of the surfaces of the Moon and Mercury with their exospheric atmospheres. Reviews of Geophysics and Space Physics 15: 491-499.

Shkrob, I.A., Chemerisov, S.D. \& Marin, T.W., 2010. Photocatalytic decomposition of carboxylated molecules on light-exposed Martian regolith and its relation to methane production on Mars. Astrobiology 10: 425436.

Shkrob, I.A., Marin, T.M., Adhikary, A. \& Sevilla, M.D., 2011. Photooxidation of nucleic acids on metal oxides: Physicochemical and Astrobiological Perspectives. Journal of Physical Chemistry C 115: 3393-3403.
Shock, E.L., 1990. Geochemical constraints on the origin of organic-compounds in hydrothermal systems. Origins of Life and the Evolution of the Biosphere 20: $331-367$

Shock, E.L. \& Schulte, M.D., 1990. Amino-acid synthesis in carbonaceous meteorites by aqueous alteration of polycyclic aromatic hydrocarbons. Nature 343: 728-731.

Stalport, F., Coll, P., Szopa, C., Cottin, H. \& Raulin, F., 2009. Investigating the photostability of carboxylic acids exposed to Mars surface ultraviolet radiation conditions. Astrobiology 9: 543-549.

ten Kate, I.L., 2010. Organics on Mars? Astrobiology 10: 589-603.

ten Kate, I.L. \& Motamedi, R., 2015. Celestial bodies. In: Beysens, D. \& van Loon, J. (eds): Generate an Extra-Terrestrial Environment on Earth. River Publishers (Aalborg): 25-35

ten Kate, I.L., Garry, J.R.C., Peeters, Z., Quinn, R., Foing, B. \& Ehrenfreund, P., 2005. Amino acid photostability on the Martian surface. Meteoritics \& Planetary Science 40: 1185-1193.

ten Kate, I.L., Garry, J.R.C., Peeters, Z., Foing, B. \& Ehrenfreund, P., 2006. The effects of Martian near surface conditions on the photochemistry of amino acids. Planetary and Space Science 54: 296.

Vasavada, A.R., Paige, D.A. \& Wood, S.E., 1999. Near-surface temperatures on Mercury and the Moon and the stability of polar ice deposits. Icarus 141: 179-193.

Zahnle, K., Schaefer, L. \& Fegley, B., 2010. Earth's earliest atmospheres. In: Deamer, D. \& Szostak, J.W. (eds.): The Origins of Life. Cold Spring Harbor Perspectives in Biology. Cold Spring Harbor Laboratory Press (Cold Spring Harbor): p. a004895.

Zolensky, M. \& McSween, H.Y.J., 1988. Aqueous alteration. In: Kerridge, J.F. \& Matthews, M.S. (eds): Meteorites and the Early Solar System. University of Arizona Press (Tucson): 114-143. 